\title{
Impact of premorbid Frailty on stroke outcomes
}

\section{R. Sivakumar, R. Saksena}

Colchester General Hospital, Stroke unit, Colchester, United Kingdom

\section{Background and Aims:}

Frailty amongst stroke patients is increasing and is associated with poorer outcomes. We sought to evaluate the impact of frailty on stroke outcomes in our population which has higher frailty rates than the national average.

\section{Method:}

\section{Design:}

Prospective cohort analysis. Stroke subjects admitted over a 4 year period in a district general hospital in UK were included. Data collected prospectively of all patients with stroke was used for analysis.

Frailty was defined as prestroke modified Rankin score of 3 and above(3-5)

\section{Results:}

Frail patients present with slightly higher severity of stroke, and are slightly less likely to receive thrombolysis. They have a much higher incidence of stroke related complications such as pneumonia, and Urinary tract infections. They are more likely to be placed on palliative care. There is a higher likelihood of death and new institutionalisation.

Key results are presented in Table 1.

Table 1

Total number of strokes

2287

4 year period

\begin{tabular}{|l|c|c|c|c|c|}
\hline & Modified Rankin score 3-5 & MRS 0-2 & Modified Rankin score 3-5 & MRS 0-2 & \\
\hline n & 728 & 1559 & & & \\
\hline Median NIHSS & 9 & 5 & $\%$ & $\%$ & p value \\
\hline Thrombolysis & 81 & 247 & 11.1 & 15.8 & 0.0028 \\
\hline UTI & 58 & 45 & 8 & 2.9 & $<0.0001$ \\
\hline Pneumonia & 89 & 113 & 12.2 & 7.2 & 0.0001 \\
\hline Palliative & 145 & 77 & 19.9 & 4.9 & $<0.0001$ \\
\hline Died & 202 & 183 & 27.7 & 11.7 & $<0.0001$ \\
\hline New Instituitionalisation & 63 & 47 & 8.7 & 3 & $<0.0001$ \\
\hline
\end{tabular}

Premorbid Rankin score of 3-5 association with increased mortality was retained(Odds ratio: 1.5934 (1.1978 to 2.1196) when corrected for other covariates such as stroke type, NIHSS, age, gender, history of Atrial Fibrillation, heart failure, Diabetes. So was the association for UTI, palliative care on discharge and new institutionalisation.

\section{Conclusion:}

Frail patients present with slightly higher severity of stroke, slightly less likely to receive thrombolysis, have a much higher incidence of stroke related complications including death and higher likelihood of new institutionalisation. By being aware of the association with pre-stroke frailty and poor outcomes, clinicians can improve outcomes by augmenting evidence based care provision for frail patients. 